\title{
Model calculations of the planetary ion distribution in the Martian tail
}

\author{
Herbert Lichtenegger ${ }^{1,4}$ and Eduard Dubinin ${ }^{2,3,4}$ \\ ${ }^{1}$ Space Research Institute, Austrian Academy of Sciences, Inffeldgasse, 12, 8010, Graz, Austria \\ ${ }^{2}$ Max-Planck-Institut für Aeronomie D-37191 Katlenburg-Lindau, Germany \\ ${ }^{3}$ Space Research Institute, Russian Academy of Sciences, 117810 Moscow, Russia \\ ${ }^{4}$ International Space Science Institute (ISSI), Hallerstrasse 6, 3012 Bern, Switzerland
}

(Received August 4, 1997; Revised March 5, 1998; Accepted April 9, 1998)

\begin{abstract}
Based on a recent model of the Martian atmosphere/exosphere and a model of the magnetic field and solar wind flow around Mars, the distribution of different planetary ion species in the near tail is calculated. Three main regions are identified: 1) "clouds" of pickup ions with distinct mass separation travel along cycloidal trajectories; 2) another group of ions forms a distinct plasma mantle in the magnetosphere; 3) a third population fills up the plasma sheet. Further, the energy of ions in different locations is also analyzed. Finally, comparison of observations made onboard the Phobos-2 spacecraft shows a reasonable agreement with simulation results.
\end{abstract}

\section{Introduction}

Measurements carried out on the Phobos-2 spacecraft have shown that the plasma tail of Mars consists mainly of ions of planetary origin (Lundin et al., 1989a, b; Rosenbauer et al., 1989). Lundin et al. (1989a, b, 1990) estimated that the planet is losing oxygen at a rate of about $3 \times 10^{25}$ ions/ s. This value is sufficiently high to explain a dehydration of Mars on a cosmogonic time scale. Based on TAUS spectrometer data, Verigin et al. (1991) have considered the distribution of heavy ions through the cross-section of the magnetotail. The average loss rate through the plasmasheet was evaluated as $5 \times 10^{24} \mathrm{~s}^{-1}$, with a typical flux of about 2.5 $\times 10^{7} \mathrm{~cm}^{-2} \mathrm{~s}^{-1}$, and they came to the conclusion that only the thermal oxygen population, which is much more abundant below the boundary of the magnetosphere, needs to supply the plasmasheet. They assumed that wide polar caps as part of the intrinsic Martian magnetosphere might be channels for the oxygen transport into the tail. Lundin et al. (1990) argued that heavy planetary ions fill the magnetotail from sides, forming a boundary layer. Using a combined model for the draping magnetosphere, Lichtenegger and Dubinin (1998) have traced the path of oxygen ions originating at different locations near Mars. It was shown that the main outflux came from low altitudes $(\approx 300-350 \mathrm{~km})$, focussing to a rather narrow plasma sheet. The values of ions fluxes in the plasma sheet were in reasonable agreement with observations. Kallio et al. (1995) have studied the distribution of $\mathrm{O}^{+}$-ions with different energies in the Martian magnetotail. They observed a steady population of oxygen ions in the shadow of the planet and attributed it to the asymmetric and dynamic plasmasheet. Unfortunately, the statistics of these measurements was not enough to get a reliable pattern of the ion distribution in the tail. Dubinin et al. (1996) have also utilized the ASPERA ion composition measurements to

Copy right $(C)$ The Society of Geomagnetism and Earth, Planetary and Space Science (SGEPSS); The Seismological Society of Japan; The Volcanological Society of Japan; The Geodetic Society of Japan; The Japanese Society for Planetary Sciences. describe the statistical morphology of ion fluxes in the magnetotail. They have analyzed the data from the masssensor of ASPERA, being able to distinguish reliably between different ions. Planetary ions fill mainly two regions in the tail, the boundary layer or mantle and the plasma sheet. However, to achieve a better statistics in their "image" of the plasma environment, they were forced to summarize all planetary ions without mass separation.

The ASPERA ion composition spectrometer made it possible to measure the distribution of different ion species. It was shown (Lundin et al., 1990; Norberg et al., 1993) that the inner magnetosphere of Mars is filled not only by $\mathrm{O}^{+}$-ions but contains also a substantial admixture of molecular ions $\left(\mathrm{O}_{2}{ }^{+}, \mathrm{CO}_{2}{ }^{+}\right)$. Cold $\mathrm{H}^{+}$-ions of planetary origin with densities of about $1-2 \mathrm{~cm}^{-3}$ were observed inside the magnetosphere, too. Dubinin et al. (1993b) have recorded two proton populations which were clearly distinguished from the energy spectra. The flux of "cold" protons, which was attributed to the planetary source, increased abruptly at the bow shock. Then, in the magnetosheath, a gradual thermalization of both components provided their mixing and a separation of both populations became less evident. Barabash and Norberg (1994) reported also the detection of planetary $\mathrm{He}^{+}$ions. Helium in the atmospheres of terrestrial planets is released from radionuclei and the value of its degassing and nonthermal loss rate may provide important information about the planet's interior. The observed density of pickup $\mathrm{He}^{+}$detected by ASPERA was $0.02-0.1 \mathrm{~cm}^{-3}$ and reached $0.2-0.7 \mathrm{~cm}^{-3}$ in the plasma sheet. Barabash et al. (1995) estimated the total $\mathrm{He}^{+}$outflow rate as $(1.2 \pm 0.6) \times 10^{24}$ $\mathrm{s}^{-1}$. According to remote measurements of the He $584 \AA$ airglow intensity on Mars made by Krasnopolsky et al. (1994), the helium loss rate is about a factor 4-5 less. Later, Krasnopolsky and Gladstone (1996) revised this value and derived the value of the total escape rate as $(7.2 \pm 3.6) \times 10^{23}$ $\mathrm{s}^{-1}$. In this paper we calculate the total escape rate and spatial downstream distribution of various atmospheric species based on a model which incorporates an MHD approach in 
the magnetosheath and a cometary like magnetotail. Another interesting aspect is the energetics of planetary ions in the tail. A typical feature of the tail crossings at $2.8 \mathrm{R}_{\mathrm{M}}$, that the energy of $\mathrm{O}^{+}$-ions in the plasma sheet is about the energy of protons in the solar wind, was reported by Dubinin et al. (1993a). These authors have shown that the acceleration of planetary ions may be explained by magnetic shear stresses of the draped magnetic field lines. Their estimates gave a reasonable agreement with observations and allowed to explain even variations in the peak energy of oxygen ions. The important feature of the Martian magnetosphere is a large Larmor radius of pickup heavy ions as compared with the width of the plasma sheet. Therefore, ions must be treated as nonmagnetized and their motion is mainly determined by the ambipolar electric field (the fluid of electrons is accelerated by the magnetic stresses and provides an ambipolar electric field which pulls the ions). Lichtenegger et al. (1995) have modeled the behaviour of ions for cases without and with this electric field. They found a much better agreement with observations taking into consideration the force associated with magnetic field stresses.

In this report we utilize the similar approach (Lichtenegger et al., 1995, 1997; Lichtenegger and Dubinin, 1998) to describe the morphology and main characteristics of ion fluxes in the Martian tail in more detail.

\section{The Combined Modelling}

The field and flow environment of Mars in numerically calculated by means of the gasdynamic model of Spreiter and Stahara (1980), which gives a solution throughout the magnetosheath. Inside the impenetrable obstacle, the field is assumed to be of a cometary tail like structure and is represented by simple analytical expressions similar to those given by Wallis and Johnstone (1983). The latter model is fit to the gasdynamic solution and a fairly smooth transition from the magnetosheath into the tail is achieved by giving the obstacle the shape of an appropriate fieldline. Identifying the observed planetpause (i.e. the boundary of the termination of the solar wind fluxes) with the obstacle in the gasdynamic code, this model of the Martian plasma environment fairly well agrees with the Phobos-2 measurements (see Lichtenegger et al., 1995).

Models of this kind have been introduced first by Luhmann (1990) and Luhmann and Schwingenschuh (1990) to simulate the plasma environment of Mars. Although the combined model has weaknesses (e.g. a somewhat unphysical boundary between the magnetosheath and tail part) and cannot correctly describe the transport of fieldtubes from the polar region into the near wake (see Tanaka, 1993), it can serve as a simple representation of the near Martian space.

Figure 1 illustrates the plasma streamlines and field lines of the magnetosheath and induced tail model in the plane of the interplanetary magnetic field (IMF) for a perpendicular upstream magnetic field. After having established the invariable background fields, test particles were launched and their trajectories followed by numerically solving the Lorentz equation of motion with gravity included. Two different cases were considered: with the motional electric field $\boldsymbol{E}=$ $-1 / c(\boldsymbol{v} \times \boldsymbol{B})$, and with additional contribution of the ambipolar electric field caused by the tension of draping magnetic field lines (Dubinin et al., 1993a; Lichtenegger et al., 1995). The space above the dayside of Mars is divided into a number of small spherical volume elements, their centers being separated by $\Delta r \cong 0.035 \mathrm{R}_{\mathrm{M}}$ and $\Delta \Theta=\Delta \varphi \sim 1^{\circ}$, where $1.09 \mathrm{R}_{\mathrm{M}} \leq$ $r \leq 2 \mathrm{R}_{\mathrm{M}}, 0^{\circ} \leq \Theta \leq 180^{\circ}$ and $-110^{\circ} \leq \varphi \leq 110^{\circ}$. One particle is launched from each center and traced several planetary radii downstream. From this motion of the particles, the relevant physical quantities are obtained in the following way. Each particle is assumed to represent all particles from its volume element, hence the number of particles per unit of time emerging from a volume element $\Delta V_{i}$ is $\Delta N_{i} / \Delta t=n_{i} \tau \Delta V_{i}$, where $n_{i}$ is the atmospheric/exospheric density within $\Delta V_{i}$ and $\tau$ is the ionization rate. The mean flux of ions originating from photoionization, impact ionization and charge exchange is calculated at several locations downstream of the planet by dividing the plane perpendicular to the sun-planet line (yz-plane) into a number of small area elements $(\Delta y=\Delta z \approx$ $\left.0.04 \mathrm{R}_{\mathrm{M}}\right)$ and adding the number of particles crossing each element: $\Phi_{x}=\sum \Phi_{x}{ }^{i}=\sum \Delta N_{i} / \Delta t \Delta y \Delta z$. The sum runs over the index " $i$ " which labels those particles $N_{i}$, which cross the respective area element. The number density is obtained via

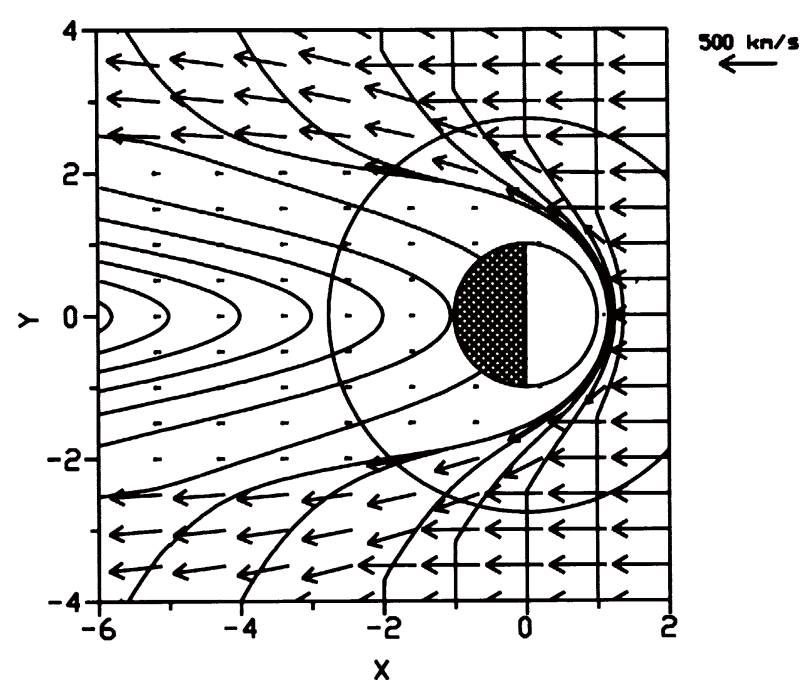

Fig. 1. Magnetic field lines and vectors of the proton bulk velocity in the $X Y$-plane of the combined magnetosheath-tail model.

Table 1. Photoionization frequencies and cross sections for different ionization processes and different exospheric species. The two entries for photoionization correspond to solar minimum and maximum, respectively.

\begin{tabular}{lllll}
\hline & \multicolumn{1}{c}{$\mathrm{H}$} & \multicolumn{1}{c}{$\mathrm{H}_{2}$} & \multicolumn{1}{c}{$\mathrm{H}$} & \multicolumn{1}{c}{$\mathrm{O}$} \\
\hline Photoionization $\left(\mathrm{s}^{-1}\right)$ & $2.9 \cdot 10^{-8} / 5.7 \cdot 10^{-8}$ & $3.2 \cdot 10^{-8} / 6.5 \cdot 10^{-8}$ & $4.3 \cdot 10^{-8} / 8.7 \cdot 10^{-8}$ & $1 \cdot 10^{-7} / 3 \cdot 10^{-7}$ \\
Charge-exchange $\left(\mathrm{cm}^{2}\right)$ & $1.7 \cdot 10^{-15}$ & $3.5 \cdot 10^{-16}$ & $2.2 \cdot 10^{-19}$ & $8.0 \cdot 10^{-16}$ \\
Electron impact $\left(\mathrm{cm}^{2}\right)$ & $7.5 \cdot 10^{-17}$ & $1.15 \cdot 10^{-16}$ & $3.34 \cdot 10^{-17}$ & $1.0 \cdot 10^{-16}$ \\
\hline
\end{tabular}


$n=\sum\left(\Delta \Phi_{x}^{i} / \Delta\left|v_{x}{ }^{i}\right|\right)$ and finally the $x$-component of the bulk velocity is given by $v_{x}=\Phi_{x} / n$.

The model of the Martian exosphere for solar minimum and maximum conditions, based on exospheric temperatures of $200^{\circ}$ and $350^{\circ} \mathrm{K}$, was taken from Krasnopolsky and Gladstone (1996). All the main sources of ionization (Photoionization, electron impact and charge exchange) were taken into account. Used ionization frequencies (or cross-sections) are given in Table 1 for minimum and maximum solar activities.

\section{Escape Rates and Distribution of Planetary Ions in the nearby Tail}

Above $\sim 300 \mathrm{~km}$ the density profiles of Krasnopolsky and Gladstone (1996) can be approximated by Chamberlain and
Hunten (1987)

$$
n(r)=\frac{2 n_{0}}{\sqrt{\pi}} \gamma\left(\frac{3}{2}, \frac{h}{r}\right) \mathrm{e}^{h / r},
$$

where $r$ is measured from the planetary center, $\chi(3 / 2, h / r)$ is the incomplete gamma function and $h=G M m / k T$, with the gravitational constant $G$, the Boltzmann constant $k$, the Martian mass $M$, the atmospheric neutral mass $m$ and the exobase temperature $T$. The total escape rate of planetary ions originating either from photoionization, charge exchange or electron impact in the dayside Martian hemisphere is hence given by

Table 2. Total escape rates from the Martian dayside hemisphere for various atmospheric species, based on two different neutral density models and constant and variable obstacle, height, respectively.

\begin{tabular}{|c|c|c|c|c|c|c|c|c|c|c|c|c|}
\hline \multirow[t]{2}{*}{ Total escape rate $\left(\times 10^{24} \mathrm{~s}^{-1}\right)$} & \multicolumn{4}{|c|}{ Photoionization } & \multicolumn{4}{|c|}{ Electron impact } & \multicolumn{4}{|c|}{ Charge exchange } \\
\hline & $\mathrm{H}^{+}$ & $\mathrm{H}_{2}^{+}$ & $\mathrm{H}^{+}$ & $\mathrm{O}^{+}$ & $\mathrm{H}^{+}$ & $\mathrm{H}_{2}^{+}$ & $\mathrm{He}^{+}$ & $\mathrm{O}^{+}$ & $\mathrm{H}^{+}$ & $\mathrm{H}_{2}^{+}$ & $\mathbf{H E}^{+}$ & $\mathrm{O}^{+}$ \\
\hline Sol. $\min .\left(R_{i}=300 \mathrm{~km}\right)$ & 3.8 & 2.2 & 0.1 & 0.2 & 18 & 28 & 0.6 & 0.8 & 26 & 2.9 & $\leq 10^{-5}$ & 0.2 \\
\hline Sol. $\max .\left(R_{i}=300 \mathrm{~km}\right)$ & 0.6 & 3.5 & 0.1 & 6.9 & 1.1 & 13 & 0.3 & 8.7 & 1.9 & 2.2 & $\leq 10^{-5}$ & 1.9 \\
\hline Sol. min. $\left(300 \leq R_{i} \leq 1300 \mathrm{~km}\right)$ & 3.3 & 1.1 & 0.03 & 0.02 & 17 & 20 & 0.3 & 0.1 & 23 & 1.6 & $\leq 10^{-5}$ & 0.01 \\
\hline Sol. $\max .\left(300 \leq R_{i} \leq 1300 \mathrm{~km}\right)$ & 0.5 & 2.9 & 0.04 & 0.8 & 1.2 & 12 & 0.2 & 2.1 & 1.8 & 1.9 & $\leq 10^{-5}$ & 0.2 \\
\hline
\end{tabular}

Density

Exospheric model for solar maximum

Ion source: Photoionization, electron impact, charge exchange
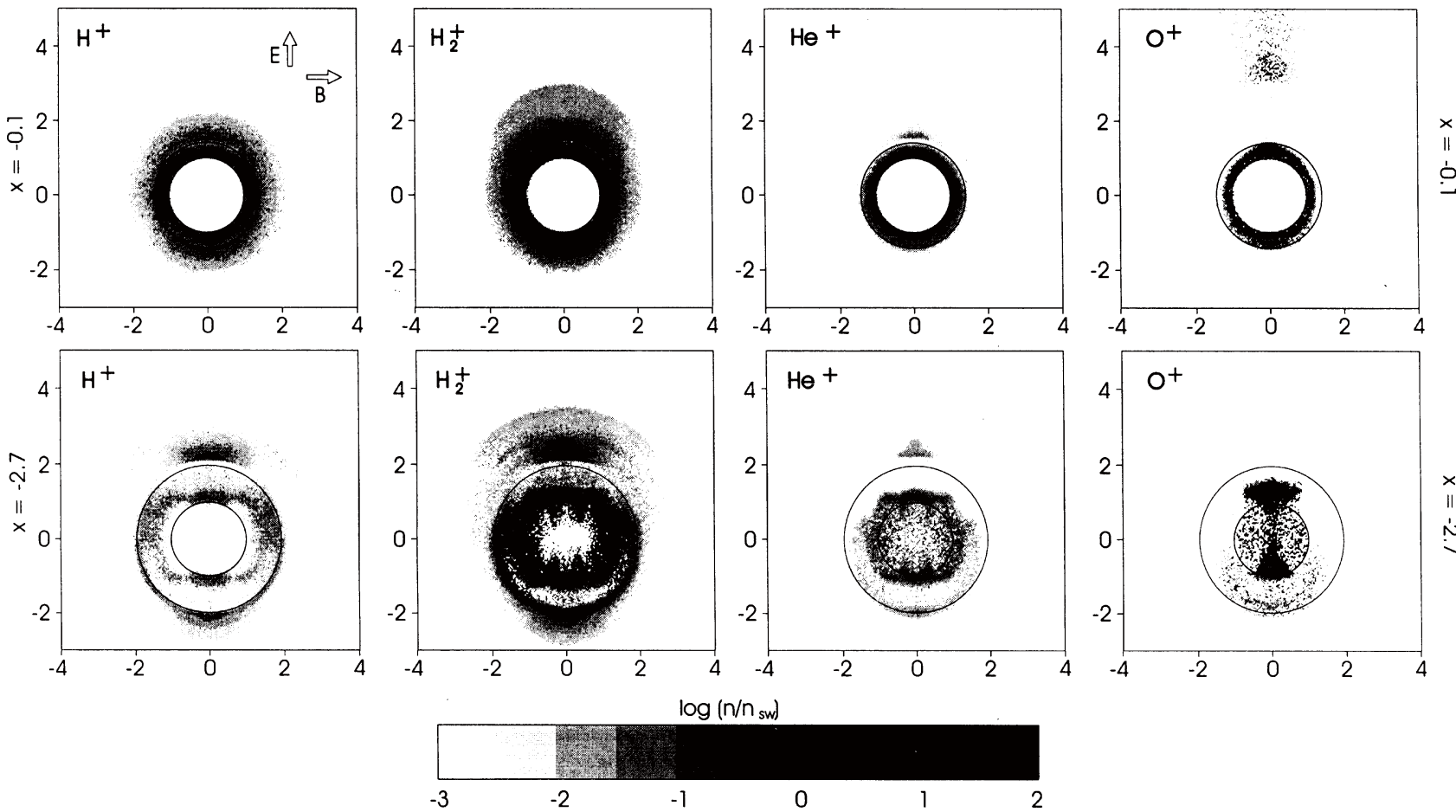

Fig. 2. Distribution of number density of planetary ion species $\left(\mathrm{H}^{+}, \mathrm{H}_{2}{ }^{+}, \mathrm{He}^{+}\right.$and $\left.\mathrm{O}^{+}\right)$in two different planes perpendicular to the Sun-Mars line, located at $x=-0.1$ and $x=-2.7 \mathrm{R}_{\mathrm{M}}$ downstream of the planet in the $\boldsymbol{v} \boldsymbol{B}$ reference frame. The inner circle represents the planet, the outer circle shows the obstacle boundary used in the simulation. 


$$
\Phi=\int \tau(r, \Theta, \phi) n(r) d V
$$

where $d V$ is the volume element in spherical coordinates and $\tau$ the position dependent ionization frequency. Equation (1) was solved numerically, where the ionization frequencies were evaluated by means of the model fluxes and the integration volume was bound by $R_{i} \leq r \leq h, 0 \leq \Theta \leq \pi$ and $-\pi / 2 \leq \phi \leq \pi / 2, R_{i}$ being the obstacle height measured from the planetary center. Calculations were performed for two different shapes of the obstacle: one with constant height of $300 \mathrm{~km}$ on the dayside and one with changing altitude from $300 \mathrm{~km}$ at the subsolar point to $\sim 1300 \mathrm{~km}$ at the terminator. Overall escape rates for different ion species and different density models for all considered ionization mechanism for the two shapes of the obstacle are summarized in Table 2. The total escape rate for helium during solar minimum is found to be $\sim 0.7 \times 10^{24} \mathrm{~s}^{-1}$ for a constant obstacle height of $300 \mathrm{~km}$ and closely agrees with the value $\sim 1.1 \times 10^{24} \mathrm{~s}^{-1}$ determined by Krasnopolsky and Gladstone (1996) if one takes into account that their value also includes the escape on the nightside. Note, however, that our value is derived by using a position dependent electron impact ionization frequency, whereas the Krasnopolsky and Gladstone (1996) number is based on a fixed frequency. Also, in contrast to us they took their solar mean model as a basis $(T=280 \mathrm{~K})$.
Figure 2 shows the density distribution of several ion species in two different planes perpendicular to the SunMars line and located at $x=-0.1$ and $x=-2.7 \mathrm{R}_{\mathrm{M}}$ downstream of the planet in the $\boldsymbol{v} \boldsymbol{B}$ reference frame (the $X$-axis is directed to the Sun, the $Y$-axis is along the perpendicular component of the IMF, and the $Z$-axis along the motional electric field $\boldsymbol{E}=-1 / c(\boldsymbol{v} \times \boldsymbol{B})$. The electric field in the simulation box was assumed to be purely motional. Close to the terminator, the exospheric protons reside within a ring (width $\sim 1 \mathrm{R}_{\mathrm{M}}$ ) just above the planet, showing only a weak preference to move into the direction of the electric field. Ring-like distributions are observed also for other ions. With an increase of the ratio $M / q$ ( $M$ and $q$ are the mass and the ion charge respectively) the width of a ring narrows. This width is apparently related with the scale height of the density profiles for atmospheric neutrals. The topmost bunch of oxygen ions following cycloidal trajectories is also observed. Farther downstream, at $x=-2.7 \mathrm{R}_{\mathrm{M}}$ the rings become more asymmetrical. This asymmetry increases for more heavy ions. Two more or less distinct "rays" of maximum number density above the polar regions have developed. We note that the formation of tail rays, accomplished by low energetic ionospheric $\mathrm{O}^{+}$ions under the influence of a convection electric field on Venus was first reported by Luhmann (1993). While the ray structures at Venus are significantly modified by the gravitational field, the ray formation at Mars is less controlled by

\section{Energy}

Exospheric model for solar maximum

Ion source: Photoionization, electron impact, charge exchange
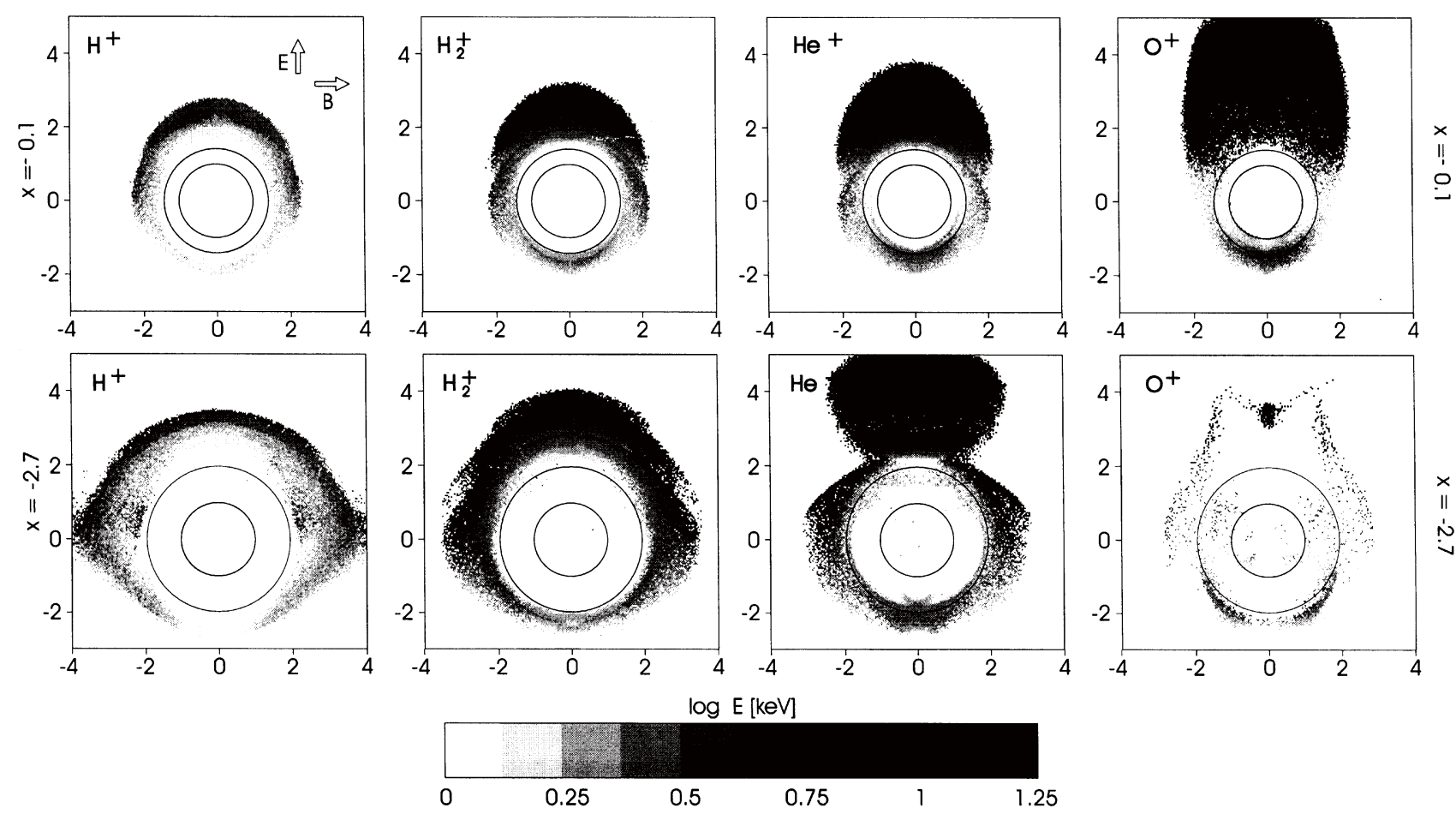

(a)

Fig. 3. Distribution of energy of planetary ion species in the same cross-sections as in Fig. 2. (a) Moderate energetic component (1-18 keV), (b) lower energy ions $\left(10^{-3}-1 \mathrm{keV}\right)$. 
gravity due to the smaller planetary mass.

The geometrical shadow of Mars is almost void of planetary protons, but mainly $\mathrm{He}^{+}$(in outlines) and $\mathrm{O}^{+}$ions occupy a concave shaped plasma sheet which separates two magnetic field lobes and extends $\sim 2-3 R_{M}$ into the direction of the electric field. Molecular hydrogen also leaks out into the shadow, giving rise to a more diffuse distribution with the tendency to form polar rays.

Thus, three main regions may be identified. "Clouds" of pickup ions with distinct mass separation travel along cycloidal trajectories. Another ion group forms a distinct plasma mantle in the magnetosphere, whereas the third population fills the plasma sheet.

\section{Ion Energetics}

Figure 3(a) and 3(b) show the energy of ion constituents in the same cross-sections as in Fig. 2. Figure 3(a) displays the more energetic component (1-18 keV). The magnetic field and motional electric field act like a huge filter separating ions with different $M / q$. The topmost bunches of ions form peculiar bulges in the northern hemisphere. Ions with larger $M / q$ populate larger bulges which gradually leave the magnetospheric cavity farther downstream. The energy of particles is increasing with $Z$, running up to several tens of $\mathrm{keV}$. Another less energetic population resides in a boundary layer/plasma mantle surrounding the magnetosphere.
Figure 3(b) presents the distribution for the energy range 1 eV-1 keV. Comparison with Fig. 2 clearly demonstrates that the energy of particles decreases toward the center and the plasma sheet consists of ions with $E \leq 100 \mathrm{eV}$. These low energy particles have small gyroradius and may drift toward the center in the draped field geometry $(\boldsymbol{E} \times \boldsymbol{B}$ drift $)$ giving rise to the plasma sheet ion population.

Dubinin et al. (1993a) have shown that magnetic shear stresses (i.e. $\boldsymbol{j} \times \boldsymbol{B}$ force) might be important for the ion dynamics. Lichtenegger et al. (1995) tried to take into consideration this force by inclusion of the Hall term to Ohm's law. The underlying idea is to consider-deviating from ideal MHD - the electron flow velocity to be different from the ion fluid velocity in the tail. In this two fluid approach, the electric field contains also terms which are proportional to the gradients of the thermal and magnetic pressure as well as to magnetic tensions related with the curvature of the fieldlines. Moreover, if different ion species are present and a differential motion of ions is admissible, as in the Martian case where the Larmor radius of heavy ions may exceed the scale of the system, additional terms in the expression for the electric field appear (see e.g. Harold and Hassam, 1994). Strictly speaking, this procedure is not compatible with the gasdynamic and tail model since it violates the assumption that the field is frozen into the flow, which is inherent in the Spreiter and Stahara approach. A more rigorous treatment,

Energy

Exospheric model for solar maximum

Ion source: Photoionization, electron impact, charge exchange
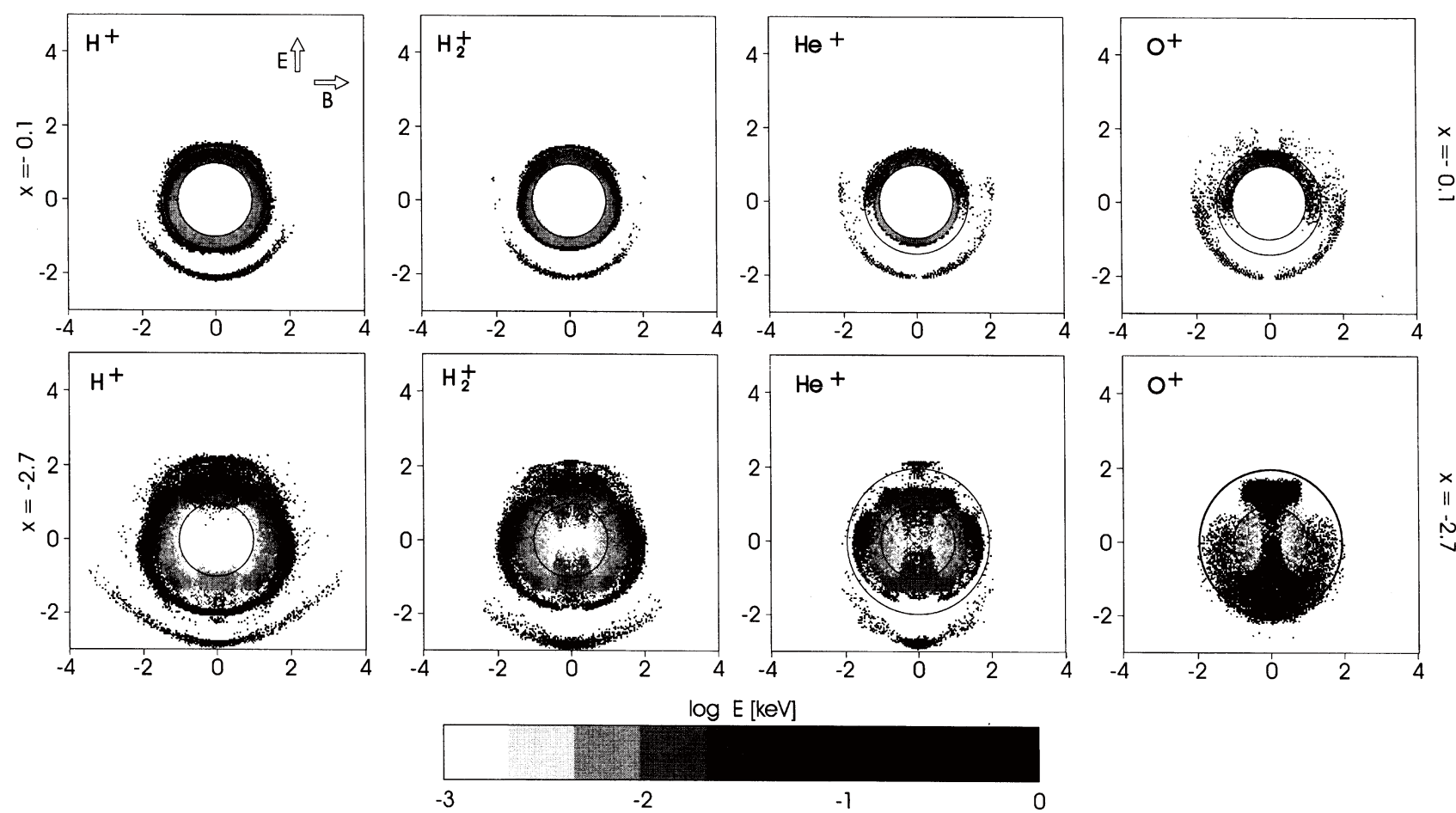

(b)

Fig. 3. (continued). 

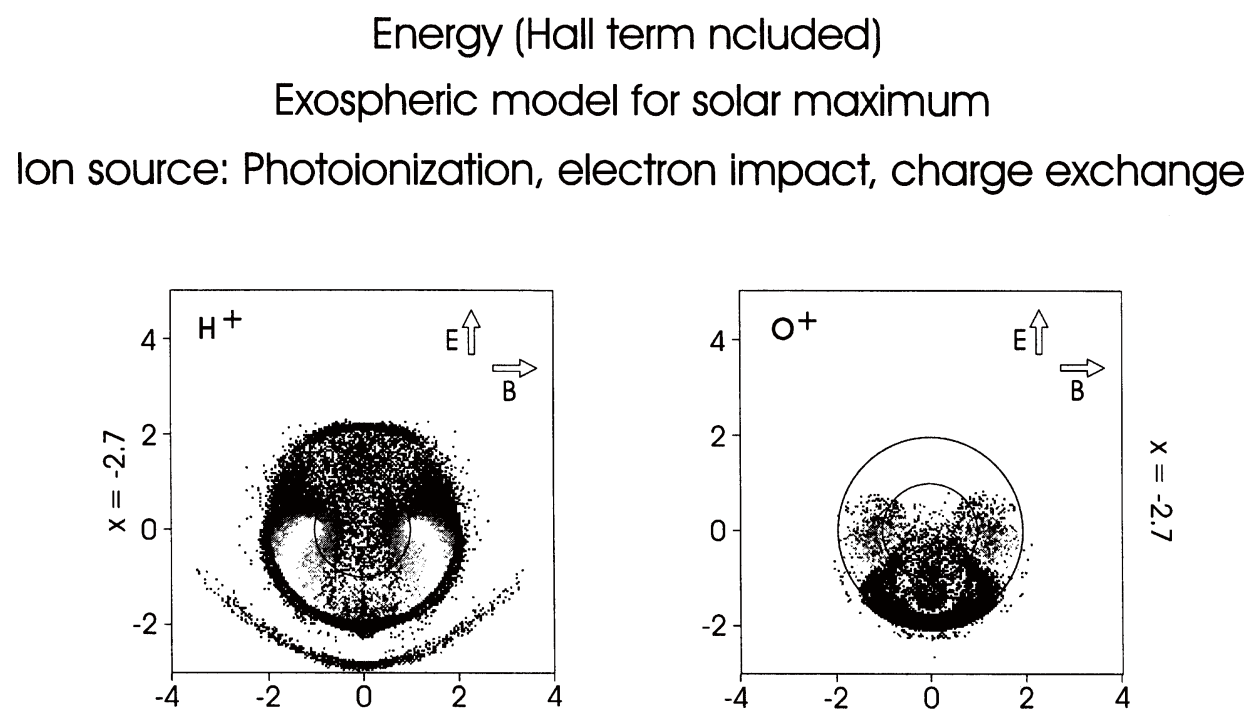

$\log E[\mathrm{keV}]$

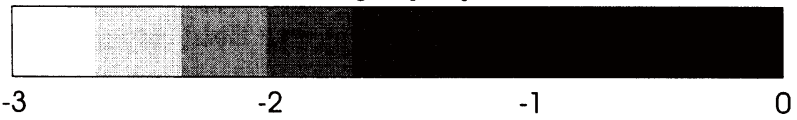

Fig. 4. Energy of $\mathrm{H}^{+}$and $\mathrm{O}^{+}$planetary ions in the tail at $x=-2.7 \mathrm{R}_{\mathrm{M}}$ for the model with inclusion of the ambipolar electric field.

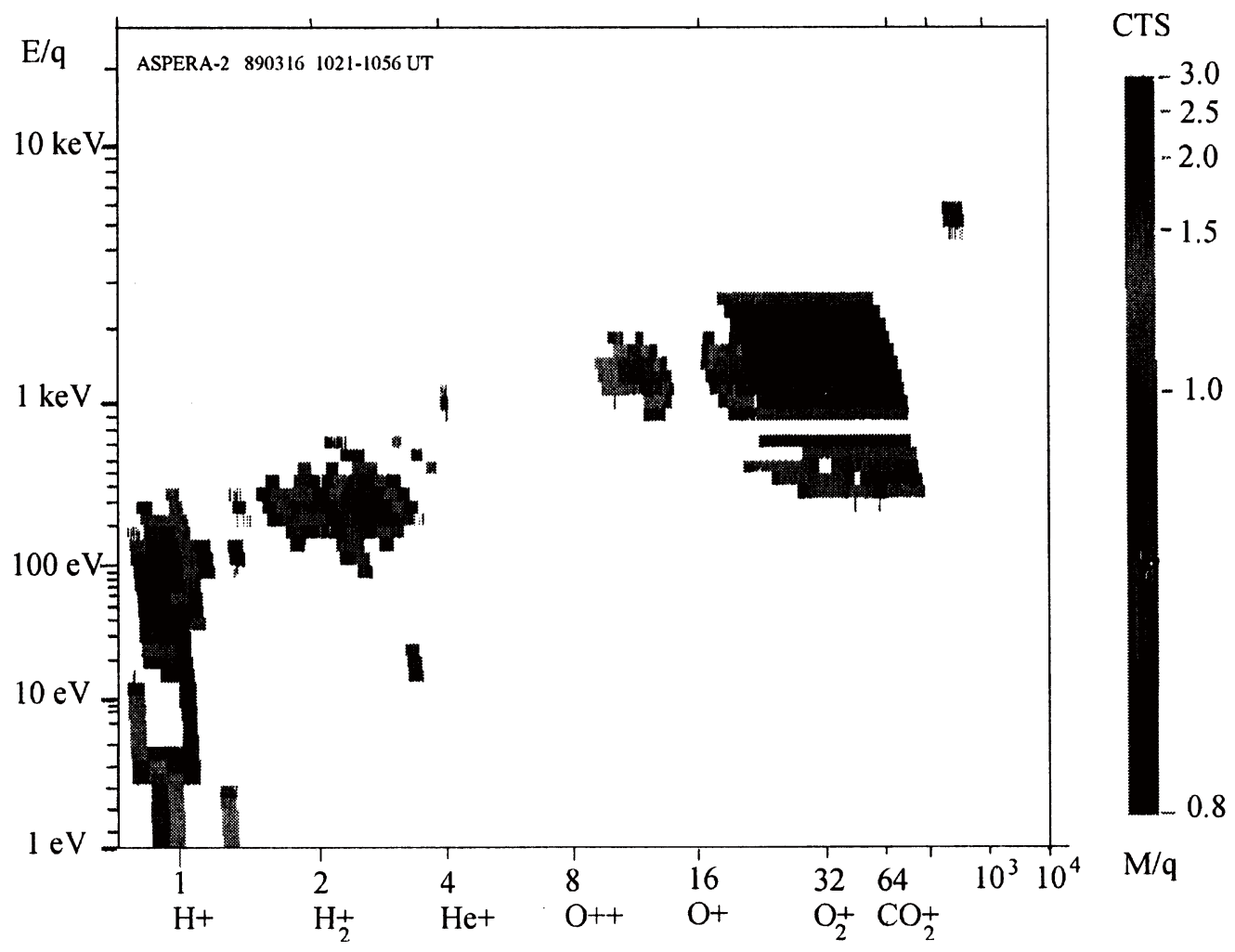

Fig. 5. Example of the ion composition in the central tail of the Martian magnetosphere. The $E / q-M / q$ matrix measured by the mass-sensor of the ASPERA instrument shows that several ion species gain different energy. 
however, requires the application of a 3-D hybrid model. Nevertheless, this modified model leads to a pattern which is in better agreement with observations made aboard the Phobos 2 spacecraft. Figure 4 illustrates the energy of $\mathrm{H}^{+}$ and $\mathrm{O}^{+}$ions at $x=-2.7 \mathrm{R}_{\mathrm{M}}$ for the modified model. The high energy part of the distribution $(E>1 \mathrm{keV})$ is not changed essentially. It is seen that, in contrast to Fig. 3(b), when approaching the center of the tail, the energy of $\mathrm{O}^{+}$ions increases to a peak value of $\sim 1 \mathrm{keV}$. Another modification, as compared with runs without the ambipolar electric field, which is also confirmed by observations, is the appearance of low energy (up to several $100 \mathrm{eV}$ ) planetary protons in the plasma sheet. These particles with a density of about $\leq 10^{-2}$ $\mathrm{cm}^{-3}$ reside within a rather broad region $\left(\sim 1 \mathrm{R}_{\mathrm{M}}\right)$.

Having in mind that comparison with observations is limited (steady state conditions and dominant cross flow component of interplanetary magnetic field needed), the model outcome is also in reasonable agreement with the results based on the analysis of high telemetry $\mathrm{O}^{+}$energy spectra obtained by the ASPERA particle detector (Kallio et al., 1995). Two of the three main $\mathrm{O}^{+}$ion populations reported by Kallio et al. (1995) are also modeled by the simulations: the shadow $\mathrm{O}^{+}$ions and the pick up $\mathrm{O}^{+}$ions. The energetic $\mathrm{O}^{+}$ions $(E>5 \mathrm{keV})$ found in the direction opposite to the motional electric field are believed to be associated with fluctuating electric and magnetic fields and cannot be reproduced within the framework of the present model.

Figure 5 presents an example of the ion composition in the central tail of the Martian magnetosphere. It shows the E/q$M / q$ matrix measured by the mass-sensor of the ASPERA instrument. The mass-sensor had a lower geometrical factor as compared with that of the "moment sensor", but was able to resolve not only major ion species $\left(\mathrm{H}^{+}, \mathrm{He}^{++}, \mathrm{He}^{+}, \mathrm{O}^{+}\right)$but also molecular ions. The energy-mass matrix $(E / q-M / q)$ was measured every $8 \mathrm{~min}$ and consisted of 32 energy levels $(E / q)$ and $128 \mathrm{M} / q$ steps in the range from $1 \mathrm{eV} / q-23 \mathrm{keV} /$ $q$ and $1-10^{4} \mathrm{amu} / q$. The field of view of the two masssensors was $5^{\circ} \times 72^{\circ}$ both in the sunward and antisunward direction. Due to the spinning of the spacecraft, sectors of $72^{\circ} \times 72^{\circ}$ were covered. The data shown in Fig. 5 were made while the spacecraft crossed the central plasma sheet $(-2.85$ $\left.\leq x<-2.63,-0.89 \leq y<0.27,0.15 \leq z<0.68 \mathrm{R}_{\mathrm{M}}\right)$. Accumulated counts in pixel were normalized to a number of sampling that varies in dependence on the pixel position in the $E / q-M / q$ matrix. More details about the instrument can be found in Lundin et al. (1989a).

Several ion species in Fig. 5 are distinctly revealed. It is assumed that the observed low energy protons are of planetary origin. Their spectrum significantly differs from the spectra of shocked solar wind protons in the magnetosheath. We also attribute ions recorded at $M / q=2$ to molecular hydrogen, because of the high ratio $n(M / q=2) / n\left(\mathrm{H}^{+}\right)$. Oxygen ions and more heavy molecular ions are evident, too. An approximately linear dependence of the ion energy with $M / q$ indicates a mechanism which accelerates ions to equal velocities. On the other hand, oxygen ions and more heavy molecular ions $\left(\mathrm{O}_{2}{ }^{+}\right)$have the same energy (energy of $\mathrm{CO}_{2}{ }^{+}$ions is even less). Dubinin et al. (1993a) suggested that the mechanism which imparts energy to the heavy ions does not depend on their mass. They assumed that these ions,
Mean Energy in plasma sheet (simulation)

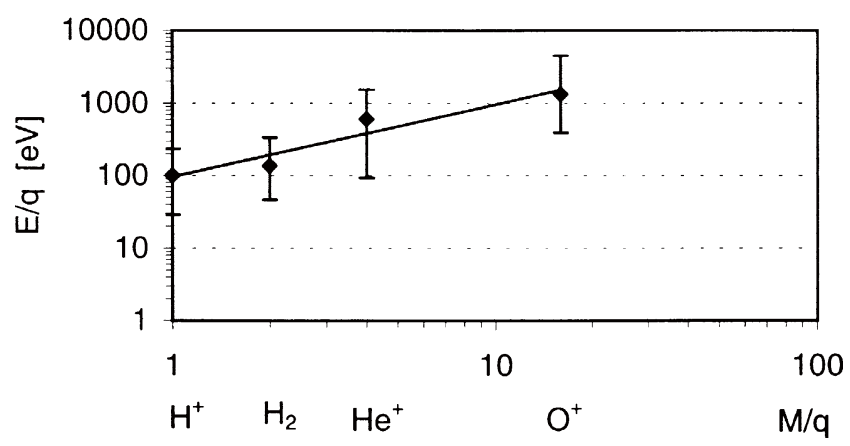

Fig. 6. Averaged energy of different ion species in the plasma sheet as a result of simulations with the modified electric field. Dark squares correspond to mean values in the area $-0.89 \leq y<0.27,0.15 \leq z<0.68 \mathrm{R}_{\mathrm{M}}$ at $x=-2.7 \mathrm{R}_{\mathrm{M}}$; bars show the standard deviation.

having large Larmor radii, are accelerated by the ambipolar electric field up to approximately the same energy per charge, while protons with smaller gyroradii gain less energy than $\mathrm{O}^{+}$. The reason for this might be seen by inspection of equation (11) from Lichtenegger et al. (1995). According to this expression, the energy $W$ particles gain due to the ambipolar electric field can be roughly estimated as $W \sim L \cdot \boldsymbol{B}^{2}$ / $(\delta \cdot n)$, where $L$ is the acceleration length, $\delta$ the gradient length of the magnetic field $\boldsymbol{B}$ and $n$ the plasma density. If the ion's gyroradius does not exceed the typical size of the system, the acceleration length is $L \sim c \sqrt{m \cdot W} / q \cdot B$. Hence, the energy $W$ is linearly proportional to the particle mass $m$. For particles with very large gyroradii (e.g. molecular ions), however, $L$ is about the size of the system and therefore $W$ does no longer depend on $m$. Probably, both mechanisms (pickup mechanism and action of magnetic field stresses) work together and give rise to a more complicated acceleration process. Figure 6 shows, for comparison, the dependence of the gained energy on the ion mass in the simulations with the modified electric field. The dark squares display ion energies averaged over the area $-0.89 \leq y \leq 0.27,0.15 \leq z \leq 0.68 \mathrm{R}_{M}$ at $x=-2.7 \mathrm{R}_{\mathrm{M}}$, thus corresponding to the same region as the ion spectra in Fig. 5; the bars represent the standard deviation of the sample. We note that these energies only weakly depend on the $x$-position, they vary by $\sim 20 \%$ in the range $-2 \leq x \leq-3 \mathrm{R}_{\mathrm{M}}$. By comparing these results with observations, a rather good agreement is evident.

\section{Summary}

Based on a recent model of the planetary exosphere and a combined model of the Martian plasma environment, the total escape rate and spatial distribution of different species $\left(\mathrm{H}^{+}, \mathrm{H}_{2}{ }^{+}, \mathrm{He}^{+}, \mathrm{O}^{+}\right)$in the nearby tail was obtained. During the Phobos 2 mission at solar maximum the total loss rate of these planetary constituents above the dayside of Mars was estimated to be $3.5 \times 10^{24}\left(\mathrm{H}^{+}\right), 1.9 \times 10^{25}\left(\mathrm{H}_{2}^{+}\right), 3.6 \times 10^{23}$ $\left(\mathrm{He}^{+}\right)$and $1.8 \times 10^{25} \mathrm{~s}^{-1}\left(\mathrm{O}^{+}\right)$. The loss rate of oxygen is within a factor of 2 in agreement with observations made by Lundin et al. $(1989 \mathrm{~b}, 1990)$, the calculated loss rate of helium is close to the value obtained by Krasnopolsky and Gladstone (1996). The observed asymmetry in flux distri- 
bution, which is controlled by the orientation of the interplanetary magnetic field, may also explain, for example, why $\mathrm{He}^{+}$-ions were observed only at $1 / 3$ of all orbits (Barabash et al., 1995). Further, the energy distribution of planetary ions is modeled and compared with ASPERA measurements. It is shown that the contribution of magnetic shear stresses is essential in explaining the observations.

Acknowledgments. The authors wish to express their gratitude to the International Institute of Space Physics (ISSI) at Bern for the support of the collaboration in the framework of the Visiting Science Programme. The comments of the referees, which helped to improve the paper are also acknowledged.

\section{References}

Barabash, S. and O. Norberg, Indirect detection of the Martian helium corona, Geophys. Res. Lett., 21, 1547-1550, 1994.

Barabash, S., E. Kallio, R. Lundin, and H. Koskinen, Measurements of the nonthermal helium escape from Mars, J. Geophys. Res., 100, 21307 21316, 1995.

Chamberlain, J. W. and D. M. Hunten, Theory of Planetary Atmospheres, 481 pp., Academic Press, 1987.

Dubinin, E., R. Lundin, O. Norberg, and N. Pissarenko, Ion acceleration in the Martian tail: Phobos observations, J. Geophys. Res., 98, 39913997, 1993a.

Dubinin, E., R. Lundin, H. Koskinen, and O. Norberg, Cold ions at the Martian bow shock: Phobos observations, J. Geophys. Res., 98, 5617$5623,1993 \mathrm{~b}$.

Dubinin, E., K. Sauer, R. Lundin, O. Norberg, J.-G. Trotignon, K. Schwingenschuh, M. Delva, and W. Riedler, Plasma characteristics of the boundary layer in the Martian magnetosphere, J. Geophys. Res., 101, 27061-27075, 1996.

Harold, J. B. and A. B. Hassam, Two ion fluid numerical investigation of solar wind gas releases, J. Geophys. Res., 99, 19325-19340, 1994.

Kallio, E., H. Koskinen, S. Barabash, C. Nairn, and K. Schwingenschuh, Oxygen outflow in the Martian magnetotail, Geophys. Res. Lett., 22, 2449-2452, 1995.

Krasnopolsky, V. A. and G. R. Gladstone, Helium on Mars: EUVE and Phobos data and implications for Mars' evolution, J. Geophys. Res., 101, 15765-15772, 1996.

Krasnopolsky, V. A., S. Bowyer, S. Chakrabarti, G. R. Gladstone, and J. S. McDonald, First measurements of helium on Mars: implications for the problem of radiogenic gases on the terrestrial planets, Icarus, 109 337-351, 1994.

Lichtenegger, H. and E. Dubinin, Solar wind absorption and loss rates of planetary ions in the Martian environment during solar minimum and maximum activity, Austrian Academy of Sciences, Report IWF9801, 1-11, 1998.

Lichtenegger, H., K. Schwingenschuh, E. Dubinin, and R. Lundin, Particle simulation in the Martian magnetotail, J. Geophys. Res., 100, 21659-21667, 1995.

Lichtenegger, H., E. Dubinin, and W.-H. Ip, The depletion of the solar wind near Mars, Adv. Space Res., 20(2), 143-147, 1997.

Luhmann, J. G., A model of the ion wake of Mars, Geophys. Res. Lett., 17, 869-872, 1990.

Luhmann, J. G., A model of the ionospheric tail rays of Venus, J. Geophys. Res., 98, 17615-17621, 1993.

Luhmann, J. G. and K. Schwingenschuh, A model of the energetic ion environment of Mars, J. Geophys. Res., 95, 939-945, 1990.

Lundin, R., B. Hultqvist, S. Olsen et al., The ASPERA experiment on the Soviet Phobos spacecraft, in Solar System Plasma Physics, Geophys. Monogr. Ser., Vol. 54, edited by J. H. Waite, R. L. Burch, and T. Moore, pp. 417-424, AGU, Washington, D.C., 1989a.

Lundin, R., A. Zakharov, R. Pellinen, H. Borg, B. Hultqvist, N. Pissarenko, E. Dubinin, S. Barabash, I. Liede, and H. Koskinen, First measurements of the ionospheric plasma escape from Mars, Nature, 341, 609-612, $1989 b$.

Lundin, R., A. Zakharov, R. Pellinen, S. Barabash, H. Borg, E. Dubinin, B. Hultqvist, and H. Koskinen, ASPERA/PHOBOS measurements of the ion outflow from the Martian ionosphere, Geophys. Res. Lett., 17, 873-876, 1990.

Norberg, O., S. Barabash, and R. Lundin, Observations of molecular ions in the Martian plasma environment, in Plasma Environment of Nonmagnetic Planets, COSPAR colloq. ser. Vol. 4, edited by T. Gombosi, pp. 299-304, Pergamon, New York, 1993.

Rosenbauer, H., N. Shutte, I. Apathy et al., Ions of Martian origin and plasma sheet in the Martian magnetosphere:L initial results of the TAUS experiment, Nature, 341, 612-614, 1989.

Spreiter, J. R. and S. S. Stahara, Solar wind flow past Venus: Theory and comparisons, J. Geophys. Res., 85, 7715-7738, 1980.

Tanaka, T., Configurations of solar wind flow and magnetic field around the planets with no magnetic field: Calculation by a new MHD simulation scheme, J. Geophys. Res., 98, 17251-17262, 1993.

Verigin, M., N. Shutte, A. Galeev, K. Gringauz et al., Ions of planetary origin in the Martian magnetosphere (Phobos-2/TAUS experiment), Planet. Space Sci., 39, 131-137, 1991.

Wallis, M. K. and A. D. Johnstone, Implanted ions and the draped cometary field, in Cometary Exploration, Vol. 1, edited by T. Gombosi, 311 pp., Central Institute for Physics, Hungarian Academy of Sciences, 1983.

H. Lichtenegger (e-mail: him@iwf.tu-graz.ac.at) and E. Dubinin (email: dubinin@helene.mpae.gwdg.de) 